\title{
Sensing Chirality with Rotational Spectroscopy
}

\section{Sérgio R. Domingos, Cristóbal Pérez and Melanie Schnell}

Deutsches Elektronen-Synchrotron, Notkestraße 85, 22607 Hamburg, Germany Christian-Albrechts-Universität zu Kiel, Institut für Physikalische Chemie, Max-Eyth-Str.1 24118, Kiel, Germany

Max Planck Institute for the Structure and Dynamics of Matter, Luruper Chaussee 149, 22761 Hamburg, Germany

Xxxx. Xxx. Xxx. Xxx. YYYY. AA:1-23

https://doi.org/10.1146/((please add article doi))

Copyright (C) YYYY by Annual Reviews. All rights reserved

\section{Keywords}

chirality, microwave spectroscopy, rotational coherence, enantiomers, diastereomers, cooled buffer gas, supersonic expansion, structure determination, high-resolution spectroscopy, population transfer

\section{Abstract}

Chiroptical spectroscopy techniques for the differentiation of enantiomers in the condensed phase are based on an established paradigm that relies on symmetry breaking using circularly polarised light. We review a novel approach for the study of chiral molecules in the gas phase using broadband rotational spectroscopy, namely microwave threewave-mixing, that is a coherent, non-linear, and resonant process. This technique can be used to generate a coherent molecular rotational signal that can be detected similarly as in conventional Fourier transform microwave spectroscopy. The structure (and thermal distribution of conformations), handedness, and enantiomeric excess of gas phase samples can be determined unambiguously employing tailored microwave fields. We discuss the theoretical and experimental aspects of the method and the significance of the first demonstrations of the technique for enantiomer differentiation and its rapid advance into a robust choice to study molecular chirality in the gas phase. Very recently, the microwave three-wave mixing approach has been extended to enantiomer-selective population transfer, an important step towards spatial enantiomer separation on the fly. 


\section{Contents}

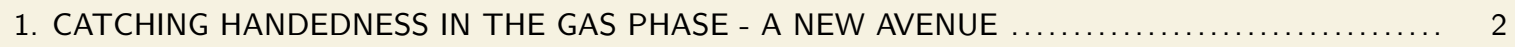

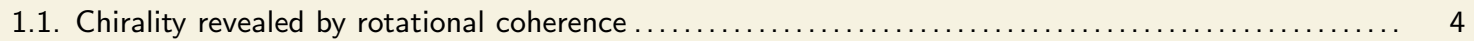

2. ENANTIOMER DIFFERENTIATION VIA MICROWAVE THREE-WAVE MIXING $\ldots \ldots \ldots \ldots \ldots \ldots \ldots$

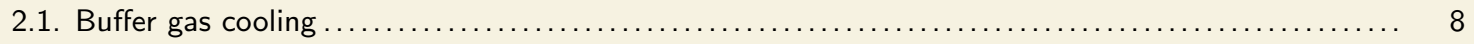

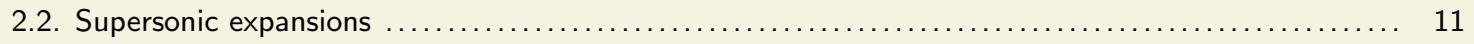

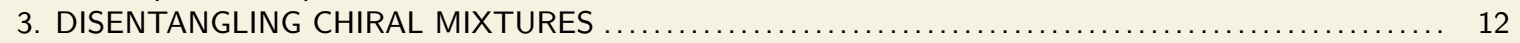

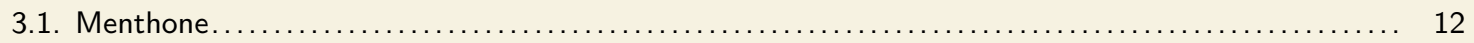

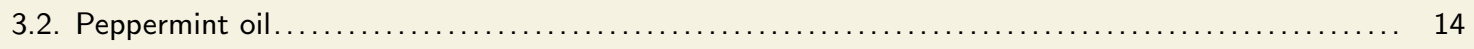

4. SHUFFLING ENANTIOMERS WITH TAILORED MICROWAVE FIELDS $\ldots \ldots \ldots \ldots \ldots \ldots \ldots \ldots \ldots \ldots$

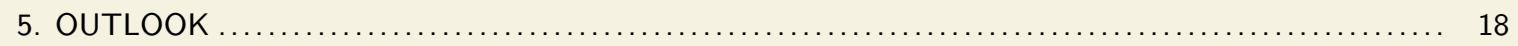

\section{Chiral}

pharmaceuticals: of the new drugs approved by the Food and Drug Administration (FDA) in 2016, more than $60 \%$ are enantioenriched.(2)

\section{CATCHING HANDEDNESS IN THE GAS PHASE - A NEW AVENUE}

Chirality plays a primordial role in (bio)chemistry. Most biomolecules are chiral, i.e., they are encoded with a preferred handedness and thus have a stereoselective bias for specific biochemical interactions. Therefore, the ability to determine the conformation and handedness of chiral molecules is crucial in establishing how molecular structure and biological activity are related. Chiroptical methods that can retrieve chiral information from a molecular system, either in isolation or in the condensed phase, are valuable tools with increasing demand in chemical and pharmaceutical sciences.

The two non-superimposable mirror images of a chiral molecule are referred to as enantiomers. The functionality of molecular systems with biological relevance is often directly related to which enantiomer is present. Therefore, a key step in understanding this functionality relies on the determination of the stereochemistry, conformation, and structural heterogeneity of these molecules. Enantiomerically pure drugs are more efficient in binding to biological targets than the equivalent racemic drugs. The reason is that our bodies consider enantiomers as different molecules, even though, apart from their stereochemistry, they have the same physical properties. This asymmetry has tremendous implications in drug development by the pharmaceutical industry (over $80 \%$ of the drugs on the market today have chiral compounds in them, and the number of enantiomerically pure drugs currently on the market is on the rise). Since the biological activity of enantiomers can be markedly different, the determination of the absolute configuration (specific handedness of an enantiomer) is an absolute requirement. Thus, fast and reliable methods that can differentiate molecules of different handedness and that can determine the enantiomeric excess (excess of one enantiomer over the other) of even molecular mixtures are of utmost importance.

Over the last decades, the chiroptical techniques available for the determination of the absolute configuration and enantiomeric excess have been based on the fact that the mirror symmetry of enantiomers can be broken using circularly-polarised electromagnetic radiation. Since the original observations of optical activity by Pasteur (1), this phenomenon has laid the grounds for established chiroptical methods used to determine the absolute configuration of chiral molecules such as optical rotary dispersion (ORD), circular dichroism (CD) (3) and vibrational optical activity (VOA) (4) methods namely vibrational circular dichroism (VCD) (5) and Raman optical activity (ROA). (6)

2 Domingos, Pérez and Schnell 
Catapulted by recent instrumental developments, a number of ground-breaking new experiments introduce alternative routes to study molecular chirality in the gas phase. Distinguishing enantiomers and measuring enantiomeric excess has been recently demonstrated, sprouting new avenues within the field of chirality-sensitive spectroscopy. Among these are Microwave Three-Wave Mixing (M3WM)(7-15), the topic of this review, Photoelectron Circular Dichroism (PECD) using intense circularly-polarised laser light or synchrotron sources (16-19) and Coulomb Explosion with Coincidence Imaging. $(20,21)$ The great precision and control that can be achieved using gas-phase samples promise a new realm for studying, controlling, and manipulating chiral molecules.

M3WM is a conceptually new method to assign the stereochemistry of chiral molecules using microwave fields that are tailored to the task, and it even provides promising prospects for manipulating and separating enantiomers. While the traditional methods are based on a mechanism that intrinsically outcomes weak signals because they rely on the interference between the electric-dipole transition moment and the weak magnetic-dipole transition moment that is detected upon irradiation with circularly-polarized light, M3WM departs from this pattern by being a fully electric-dipole allowed method. It is based on the orthogonality of the employed microwave fields that generate a third, mutually orthogonal field as a molecular response that is on the same order of magnitude than conventional Fourier-transform microwave (FTMW) spectroscopy signals, and it fulfils the requirements of a measure of true chirality (6) (see Fig. 1).

M3WM is based on high-resolution rotational spectroscopy in the gas phase.(15) Since the rotational spectra of molecules depend on their moments of inertia and thus on the distribution of atomic masses in the molecule, it is highly selective and sensitive to even only small structural or mass changes. As a consequence, isomers, conformers, and isotopologues exhibit distinct rotational spectra, like a molecular fingerprint. M3WM shares these features. These characteristics make rotational spectroscopy a powerful instrument for complex mixture analysis. (22) This became particularly applicable with the advent of broadband chirped-pulse rotational spectroscopy about a decade ago (23), pioneered by the research group of Brooks Pate (24). It significantly changed the way we perform, understand, and analyse rotational spectra. Broad sections of pure rotational spectra covering several $\mathrm{GHz}$ can be recorded in a single acquisition. Using these broadband spectra, many different species can be simultaneously and definitely identified via their rotational signatures, like up to 15 distinct conformers of citronellal (25) and hexanal (26), and numerous water clusters $\left(\mathrm{H}_{2} \mathrm{O}\right)_{n}(27,28)$ with up to $n=10$. With the extension to the millimetre range,(29) laboratory broadband measurements can also be combined with radio-telescope observations for the assignment of chemical species in the interstellar medium.(30) Additionally, metal complexes,(31, 32) plasmas,(33) flash pyrolysis reaction intermediates,(34) and Rydberg transitions (35) have also been investigated using CP-FTMW spectroscopy.

As an example, the Hamburg COMPACT spectrometer covers $6 \mathrm{GHz}$ of bandwidth in a single acquisition, employing a $4 \mu s$ long chirp from 2-8 GHz. (36) It can be easily extended to higher frequencies by only small changes in the electronic equipment. With a typical line width of $20-25 \mathrm{kHz}$, about 250.000 to 300.000 rotational transitions can be well resolved in such a $6 \mathrm{GHz}$ wide spectrum, making it highly attractive for the analysis of mixtures of even structurally very similar components, like the terpenes presented in Section 3. Stringent requirements for both rotational spectroscopy and its chirality-sensitive extension M3WM are that the molecules of interest can be brought into the gas phase and that they have a non-zero dipole moment (for M3WM, all three dipole-moment components have to be
CP-FTMW:

Chirped-pulse Fourier transform microwave spectroscopy

M3WM: Microwave Three-Wave Mixing

COMPACT: compact-passage acquired coherence technique 
non-zero). The combination of rotational spectroscopy and M3WM thus is a promising tool set for the chemical, structural, and chiral analysis of complex chiral samples, as also outlined in Section 3.

In this review, we will cover the theoretical fundamentals at play in M3WM and its experimental implementation (1.1). In Section 2 we discuss the first proof-of-concept experiments and its variants for different experimental setups employing single- (2.1.1) and double-resonance (2.1.2) approaches. In Section 3 we present recent investigations using M3WM to disentangle complex mixtures of chiral molecules in naturally occurring samples such as essential oils. More advanced implementations of M3WM to initiate quantum-state separation of enantiomers are presented and discussed in Section 4. Finally, we give an outlook for future directions, including the experimental challenges and promising targets along fundamental research lines and in a broad scope of analytical applications.

\subsection{Chirality revealed by rotational coherence}

The first experimental scheme for enantiomer differentiation using microwave spectroscopy was devised and implemented by Patterson, Schnell, and Doyle(7) using buffer-gas-cooled Sand R-enantiomers of 1,2-propanediol. The experimental details of the setup using buffergas cooling can be found elsewhere, and only the crucial aspects will be mentioned here when required. The basic idea for this experiment arises from the fact that enantiomers have an asymmetric distribution of charge that generates a total permanent dipole moment that is mirrored.(37) Whilst one can define any orientation of the inertial axes such that two of the dipole moments are positive, the orientation and thus sign of the third component will be fixed. This results in a handed coordinate system that intrinsically encodes a new measure of chirality. The quantity defined by the triple product of dipole moments changes sign under spatial inversion and is even under time-reversal symmetry. It is thus a measure of true chirality. A schematic of the axis operation is shown in Fig. 1. The projection of this quantity has opposite signs for enantiomers of a chiral molecule and is zero for any achiral molecule.

(a)

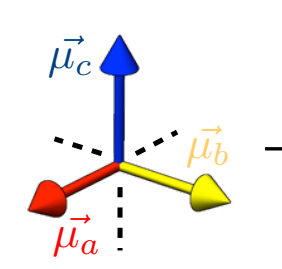

$\overrightarrow{\mu_{a}} \cdot\left(\overrightarrow{\mu_{b}} \times \overrightarrow{\mu_{c}}\right)>0$ (b)

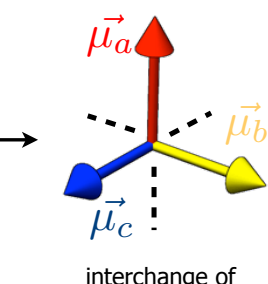
any 2 axes (c)

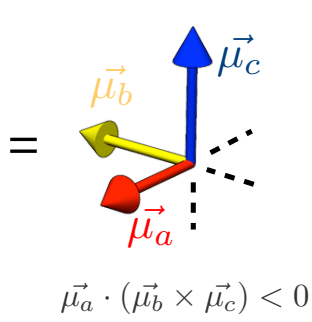

(d)

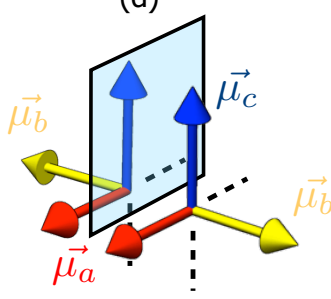

mirror reflection $(\mathrm{a} \mid \mathrm{c})$

\section{Figure 1}

Non-zero dipole moment components in a chiral molecule with $\mathrm{C}_{1}$ symmetry and its mirror image. (a) Generalised orthogonal dipole moment components $\overrightarrow{\mu_{a}}, \overrightarrow{\mu_{b}}$ and $\overrightarrow{\mu_{c}}$ with positive triple product. (b) Two components are interchanged, resulting in axis frame (c) that has a negative triple product of dipoles. (d) mirror image reflection of (a) and (c), which are enantiomers and have oppositely-signed dipole moment triple products. The triple product is odd (changes sign) under spatial inversion and is even (maintains the sign) under time-reversal symmetry, therefore qualifying as a measure of true chirality.(6)

4 Domingos, Pérez and Schnell 
So far, the method has been employed either in a buffer-gas cell, as in the original demonstration, or using the widely established supersonic expansion. Experiments at room temperature should in principle also be possible, although these have not been reported so far. The experiment can be understood as a polarisation-sensitive double-resonance experiment using a combined excitation of rotational energy levels that are connected by a-, b-, and c-type transitions (that only depend on $\mu_{a}, \mu_{b}$, and $\mu_{c}$, respectively - Fig. 1). A typical energy scheme is depicted in Fig. 2, where triads of transitions that include all three dipole-moment components are employed. Note that rotational transitions solely rely on one dipole-moment component and not on a combination thereof. For example, by first interrogating $\mu_{b}$ and $\mu_{a}$ with two orthogonal fields exciting the "drive" and the "twist" transitions (Fig. 2), the phases of the molecular signals obtained as free-induction decay (FID) in the third mutually orthogonal direction involving $\mu_{c}$ (the "listen" transition) differ by $\pi$ radians between enantiomers due to the sign change for the triple product $\vec{\mu}_{a} \cdot\left(\vec{\mu}_{b} \times \vec{\mu}_{c}\right)$

(Fig. 1). This unambiguous phase difference provides a highly sensitive measurement of chirality. A non-resonant DC field that mixes the respective energy levels can also be used instead of the second resonant twist excitation. This was the scheme originally employed by Patterson et al. (7) in the first demonstration of chirally-sensitive microwave spectroscopy.

This method allows for selective detection of enantiomeric excess using microwave spectroscopy for a wide range of chiral molecules. The molecules need to have $C_{1}$ symmetry for all three dipole moments to be non zero, and they need to be evaporated in a supersonic expansion or in a cooled buffer gas for performing the experiment in the gas phase. An unmatched advantage of this method arises from the unrivalled resolution of CP-FTMW spectroscopy, which is intrinsically able to discriminate even complex mixtures at measurement times as fast as seconds.

1.1.1. Basic theoretical framework. The time evolution of an isolated quantum system comprising three rotational energy levels of a chiral molecule can be described using the density matrix formalism.(38) The density matrix for a triad of states is a three-by-three matrix constituted by diagonal elements $\rho_{i i}(i=a, b, c)$ defining the populations of each state, whereas the off-diagonal elements $\rho_{i j}(i \neq j=a, b, c)$ describe the coherences between the states $|i\rangle$ and $|j\rangle$.

$$
\hat{\rho}=\left(\begin{array}{ccc}
\rho_{a a} & \rho_{a b} & \rho_{a c} \\
\rho_{b a} & \rho_{b b} & \rho_{b c} \\
\rho_{c a} & \rho_{c b} & \rho_{c c}
\end{array}\right)
$$

The Hamiltonian for a three microwave-pulse scheme is the sum of the unperturbed Hamiltonian $\hat{\mathcal{H}}_{0}$ and the term $\hat{\mathcal{V}}$ describing the interaction with the microwave fields. In M3WM, this interaction is induced by two incoming linearly polarised electromagnetic waves: $\mathbf{F}_{\mathbf{1}}=(1 / 2) \mathcal{E}_{1} \mathbf{e}_{x}\left(e^{i \omega t}+e^{-i \omega t}\right)$ propagating in the z-axis with amplitude $\mathcal{E}_{1}(\mathbf{r}, \mathrm{t})$, and a second, orthogonal wave $\mathbf{F}_{\mathbf{2}}=(1 / 2) \mathcal{E}_{2} \mathbf{e}_{z}\left(e^{i \omega t}+e^{-i \omega t}\right)$ with amplitude $\mathcal{E}_{2}(\mathbf{r}, \mathrm{t})$. These are the drive and twist pulses, respectively. (See Fig. 2 for relevant polarisation relations between waves.) The interaction term $\hat{\mathcal{V}}$ is given by the product of the space-fixed dipole moment operator $\hat{\mu}$ and the microwave field $\mathbf{F}=\mathbf{F}_{\mathbf{1}}+\mathbf{F}_{\mathbf{2}}$.

$$
\hat{\mathcal{H}}=\hat{\mathcal{H}}_{0}-\hat{\mathcal{V}}
$$




$$
\begin{gathered}
\hat{\mathcal{H}}_{0} \equiv\left(\begin{array}{ccc}
E_{a} & 0 & 0 \\
0 & E_{b} & 0 \\
0 & 0 & E_{c}
\end{array}\right) \\
\hat{\mathcal{V}}(t)=\hat{\mu} \cdot \mathbf{F}=\left(\begin{array}{ccc}
0 & \mu_{a b} \mathbf{e}_{x} & \mu_{a c} \mathbf{e}_{y} \\
\mu_{b a} \mathbf{e}_{x} & 0 & \mu_{b c} \mathbf{e}_{z} \\
\mu_{c a} \mathbf{e}_{y} & \mu_{c b} \mathbf{e}_{z} & 0
\end{array}\right) \cdot \mathbf{F}
\end{gathered}
$$

In the interaction region of the microwave experiment, many molecules are excited simultaneously. As such, the spectroscopic quantities evaluated refer to a statistical ensemble of quantum states. The time evolution of the density matrix is expressed by Equation 5:

$$
i \hbar \frac{\partial \hat{\rho}(t)}{\partial t}=[\hat{\mathcal{H}}(t), \hat{\rho}(t)]
$$

The expectation value for the experimentally accessible polarisation $\hat{P}$ is given by:

$$
\langle\hat{P}\rangle=N \cdot \operatorname{Tr}\{\hat{\rho} \hat{\mu}\}
$$

where $\mathrm{N}$ is the number of excited molecules and $\hat{\mu}$ is the transition dipole-moment operator. The excitation sources in the laboratory are set such that the incoming fields (drive and twist) are exclusively coupling to different transition dipole moment components $\mu_{i j}$ (with $i \neq j=a, b, c)$. The induced field (listen) can be translated as the polarisation $\hat{P}$ that is mutually orthogonal to the drive and twist fields. An expression for the listen signal can be reduced to a simple form as:

$$
\hat{P}_{\text {listen }}(t) \propto e e \cdot\left|\mu_{a} \mu_{b} \mu_{c}\right| \cdot \cos \left(2 \pi \nu t+\frac{\pi}{2} \frac{\mu_{a} \mu_{b} \mu_{c}}{\left|\mu_{a} \mu_{b} \mu_{c}\right|}\right)
$$

where $\mu_{i}(i=a, b, c)$ are the permanent dipole moment components of the chiral molecule; $\nu$ is the frequency of the listen transition and $t$ is the time interval between the beginning of the excitation and the beginning of detection. The overall signal intensity is directly proportional to the enantiomeric excess (ee). A non-zero net signal arises only if one of the enantiomers is in excess, i.e., it is a background free method. Normalising the signal amplitude with that of a sample with known $e e$ gives the $e e$ of the target sample. A full derivation of this expression has been given elsewhere (9). Because the incoming fields (drive and twist) have different arrival times at the interaction region, the phase relationships and timings between drive, twist and listen fields must be carefully taken into account for a precise determination of the absolute configuration. These aspects are explored and discussed in reference (14). Until a more robust method has been developed to precisely determine the absolute phase relationship, assignment of the absolute configuration of a chiral sample requires comparison of the measured signal with that of a sample of known chirality, as is discussed in more detail in Section 3.

1.1.2. From theory to practice. The rotational constants $A, B$ and $C$ of a chiral molecule and the corresponding dipole moment component magnitudes $\left|\mu_{a}\right|,\left|\mu_{b}\right|$ and $\left|\mu_{c}\right|$ determine the rotational energy levels of the molecule in the presence of a microwave field. The rotational transitions can be classified as purely a-, b- or c-type and the corresponding transition matrix elements $\left\langle\mu_{i j}\right\rangle$ are proportional to $\mu_{a}, \mu_{b}$ and $\mu_{c}$, respectively. The combined quantity $\vec{\mu}_{a} \cdot\left(\vec{\mu}_{b} \times \vec{\mu}_{c}\right)$ is independent of the chosen reference frame and is oppositely signed for

Domingos, Pérez and Schnell 
(a)

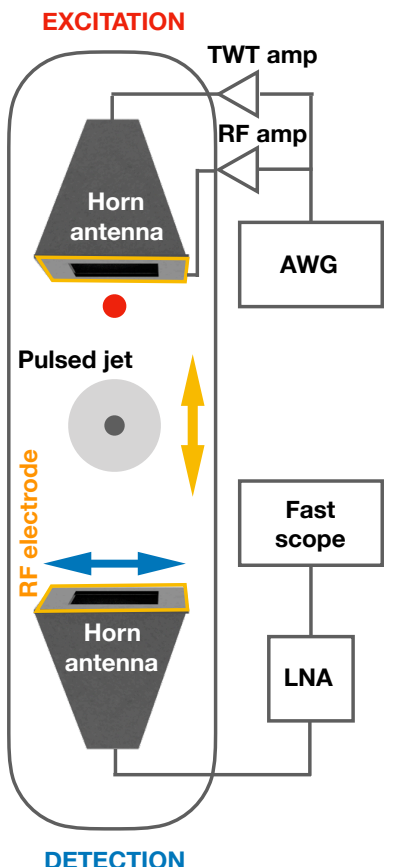

(b)
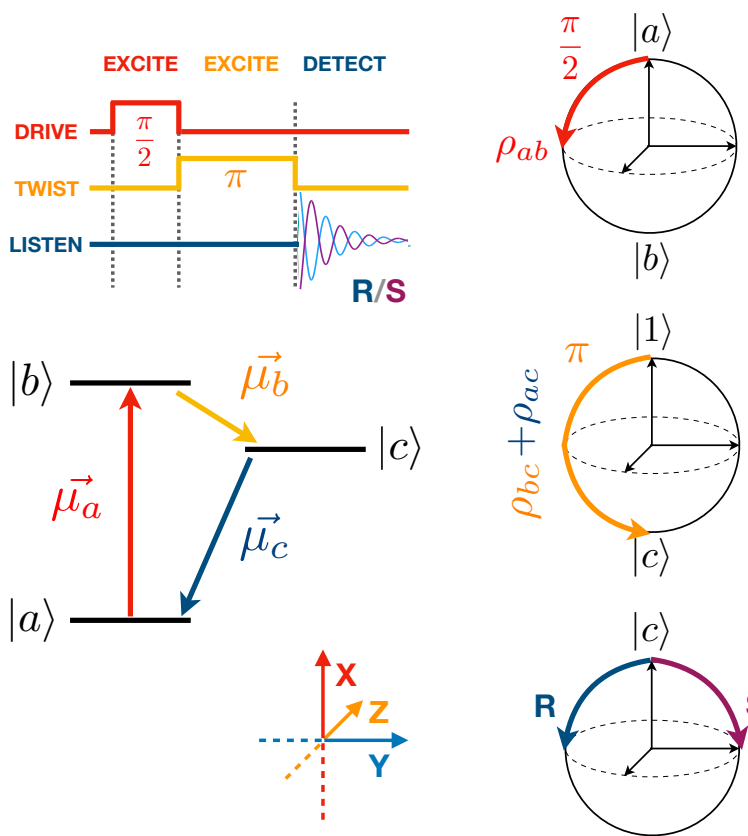

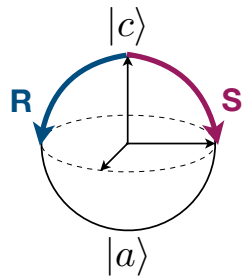

Figure 2

(a) Schematics of the Hamburg COMPACT instrument with relevant components and polarisations employed for a typical M3WM measurement. (b) Pulse sequence and energy level scheme: drive (a-type, $\pi / 2$, red excitation), twist (b-type, $\pi$, yellow excitation), and listen (c-type, blue and purple detection, enantiomer-selective); Bloch sphere representation of the M3WM scheme. The upper sphere depicts the rotational states connected via the drive transition. Upon excitation with a $\pi / 2$ pulse, a coherence is created between states $|a\rangle$ and $|b\rangle$. The middle sphere depicts the coherence transfer $\left(\rho_{a b} \rightarrow \rho_{a c}\right)$ achieved when the twist pulse is employed under $\pi$ conditions between $|b\rangle$ and $|c\rangle$. The lower sphere shows the $\pi$-shifted phase between enantiomers due to the dipole triple-product that is detected via the c-type transitions connecting states $|a\rangle$ and $|c\rangle$. The appropriate polarisations are indicated.

enantiomers. The projections of the dipole moment components on the molecular frame can be addressed independently using the applied laboratory frame microwave fields in the appropriate polarisations (see Fig. 2).

A typical rotational energy level scheme is given in Fig. 2 (b). A certain population of enantiomers is initially in the lowest energy state (bottom energy level, $|a\rangle$ ). By applying a resonant $\hat{x}$ polarised field, $E_{a}$, at frequency $\nu_{a}$ to drive the a-type transition, we generate a superposition state between $|a\rangle$ and $|b\rangle$ (see the Bloch sphere representation in Fig. 2 (b), red) with the general representation:

$$
|1\rangle=\cos \left(\frac{\Theta}{2}\right) e^{-i E(|a\rangle) t / \hbar}|a\rangle+i \sin \left(\frac{\Theta}{2}\right) e^{-i E(|b\rangle) t / \hbar}|b\rangle
$$

where $\mathrm{E}(|a\rangle)$ and $\mathrm{E}(|b\rangle)$ are the rotational energy levels of states $|a\rangle$ and $|b\rangle$, respectively, and $\Theta$ is the Rabi flip angle defined as $\frac{\langle\mu\rangle E}{\hbar} t_{\text {pulse }}$. Assuming a pure $\Theta=\pi / 2$ pulse, the 
superposition state $|1\rangle$ corresponds to the maximum coherence $\rho_{a b}$ in the density matrix (see Eq. 1 and refs. $(9,13)$ ). A second, $\hat{z}$ polarised field $E_{b}$ at frequency $\nu_{b}$ is introduced at $\Theta=\pi$ conditions to not only generate $\rho_{b c}$ coherence, but also transfer $\rho_{a b}$ into $\rho_{a c}$ coherence (Fig. 2, yellow). When applied to the molecular ensemble, this pulse sequence results in a molecular response in the form of radiation polarised along the $\hat{y}$ axis at a frequency $\nu_{c}=\nu_{a}-\nu_{b}$, which emanates solely from a c-type transition. The mutually orthogonal excitation-detection scheme ensures a readout of the portion of the polarisation that contains the chiral phase information (Fig. 2, blue and purple traces) and therefore the handedness of the enantiomer in excess: in the weak pulse limit, $E_{y}$ is proportional to $\vec{\mu}_{a} \cdot\left(\vec{\mu}_{b} \times \vec{\mu}_{c}\right)$, and it changes sign with enantiomer.

A more technical guide towards M3WM is given in Reference (14). In short, preparation of $\mathrm{M} 3 \mathrm{WM}$ experiments requires information on the volatility, the rotational energy levels, and the dipole-moment components of the sample molecules. Suitable M3WM cycles of rotational energy levels have to be identified, which have to fit into the frequency ranges that can be addressed by the chirality-sensitive spectrometers. The dipole-moment components can be evaluated using standard quantum-chemical calculations, for example, and information on the rotational energy levels and transition intensities are obtained from rotational spectroscopy. Once M3WM cycle candidates are identified, nutation curves are recorded for the drive and the twist transitions, i.e., the transition intensities are recorded as a function of the excitation pulse duration, to determine the optimal pulse durations, which correspond to Rabi $\pi / 2$ and $\pi$ conditions for the drive and the twist transition, respectively. Here, the cycles are typically arranged such that the strongest transition is chosen for the listen transition. In a more advanced scenario, these steps consisting of i) normal rotational spectroscopy, ii) M3WM cycle evaluation and optimisation, followed by iii) the actual M3WM experiments for enantiomer differentiation can be envisioned as automated procedures. $(26,39,40)$

\section{ENANTIOMER DIFFERENTIATION VIA MICROWAVE THREE-WAVE MIXING}

\subsection{Buffer gas cooling}

Enantiomer-specific detection of chiral molecules using microwave spectroscopy was firstly achieved in a buffer-gas cooling experiment.(7) Because of the continuous nature of the buffer-gas source and its controlled collisional environment, state-specific spectroscopy is possible with resolution comparable to that achieved in supersonically cooled expansions (which will be discussed in the next section). For a detailed description of the experimental aspects of a buffer gas cell for cold molecule physics experiments, the reader is referred to references (41-43). Molecular samples of R- and S-1,2-propanediol were injected at room temperature into the cryogenic buffer gas cell that is hooked to a closed-cycle pulse tube refrigerator and cooled to temperatures around $7 \mathrm{~K}$. The molecules enter the cell continuously from a warm $(300 \mathrm{~K})$ feed tube and immediately start to cool through collisions with the cold helium buffer gas. The cold molecules diffuse through the cell for several milliseconds, until they reach the cell walls and freeze there. Two distinct experimental M3WM approaches were demonstrated using the buffer-gas setup: a DC field assisted (7) and a fully resonant (8) approach. 
(a)

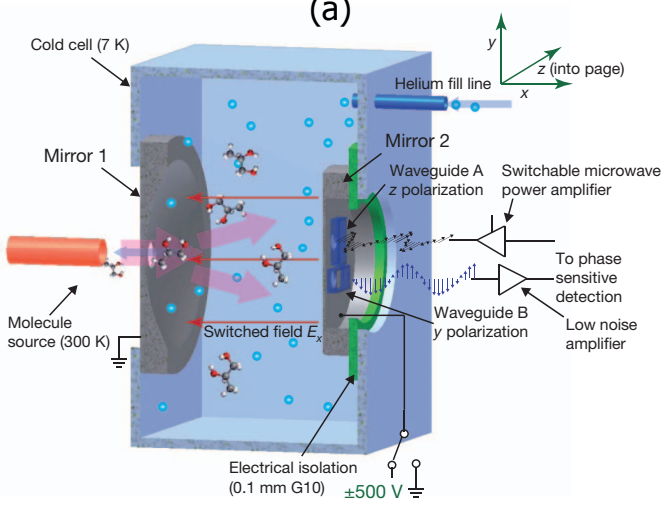

(c)

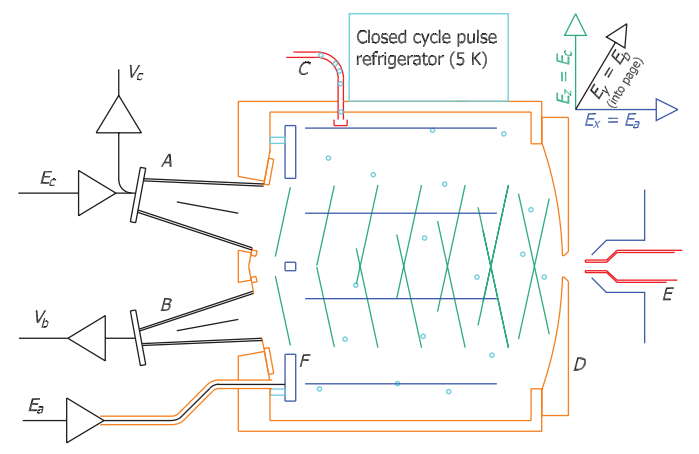

(b)

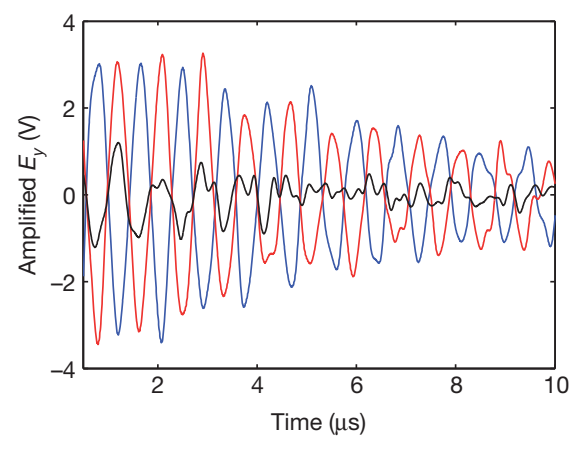

(d)

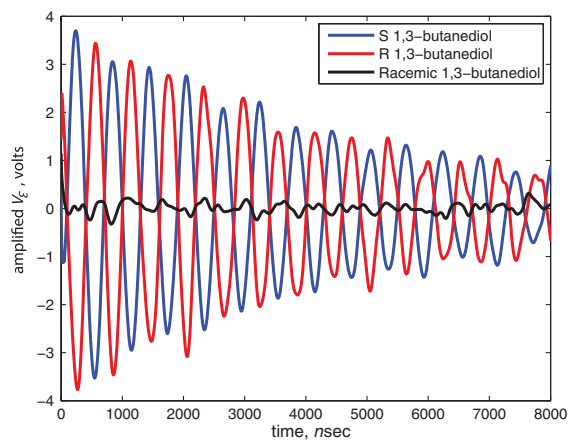

Figure 3

(a) A cryogenic buffer-gas Fourier-transform microwave spectrometer that provides enantiomer-specific detection using the DC field assisted scheme. Molecules are introduced into a cryogenic cell from a room-temperature tube held close to an aperture in mirror 1 . Microwave radiation is introduced using waveguides via coupling apertures in mirror 2 . This mirror can be rapidly switched between $6500 \mathrm{~V}$ and ground, applying a time-varying electric field in the $\mathrm{x}$ direction; it is insulated from the cell by G10 fibreglass. The molecules are polarized initially in the $\mathrm{z}$ direction via a linearly polarized microwave pulse coupled from waveguide A. The resulting enantiomer-specific radiation is coupled out of the cavity via waveguide $\mathrm{B}$, which is oriented to detect y-polarized microwaves. (b) FID of R- (red), S- (blue), and racemic 1,2-propanediol demonstrating the clear phase difference between the two enantiomers. (c) Schematics of the buffer-gas cell used for the fully resonant experiment. Here, the Fabry-Pérot type cavity is exchanged by an arrangement of horn antennas and a microwave mirror to allow for all-resonant M3WM. (d) Chiral FID signature of R-, S- and racemic 1,3-butanediol. Adapted with permission from Patterson et al. (7, 8). Copyright 2013 of Nature and American Physical Society.

2.1.1. DC field assisted scheme. Here, a Fabry-Pérot microwave cavity was built as part of a cryogenic cell. Orthogonally-polarised excitations are achieved via the transverse and longitudinal spatial cavity modes. In Fig. 3 (a) we show a schematic of the experimental setup as reported by Patterson et al. (7) using 1,2-propanediol as a test molecule.

The relevant rotational energy levels of 1,2-propanediol are given in reference (7) but it can be rendered similarly to the one given in Fig. 2 (b). Molecules initially in the lowest energy state of the scheme are prepared in a superposition of $|a\rangle$ and $|b\rangle$ using a resonant 
drive pulse, $\mathrm{E}_{x}$, with excitation frequency $\nu_{a}$ that is tuned to the $|a\rangle \rightarrow|b\rangle$ rotational transition of the ground-state conformer of 1,2-propanediol. This microwave pulse induces an oscillating electric dipole polarization, $\mathrm{P}_{x}$, in the molecular ensemble. A switched, nonresonant $\left(\mathrm{dc}\right.$ ) electric field (twist), $\mathrm{E}_{z}$, is applied parallel to the cavity axis (orthogonal to $\mathrm{E}_{x}$ ) by rapidly changing the voltage applied to the planar mirror (see Fig. 3 (a)). $\mathrm{E}_{z}$ is switched over about $100 \mathrm{~ns}$ - rapidly compared to the molecular dephasing but slowly compared to $\nu_{a}$. The variation in $\mathrm{E}_{z}$ induces a considerable fraction of the oscillating molecular dipole to radiate at the same frequency $\nu_{a}$ with $\hat{y}$-polarisation (note that the coordinate system used for describing this excitation scheme corresponds to the one in Fig. 2, to make it easier to follow, and differs from the one illustrated in Fig. 3 (a)).

The induced field $\mathrm{E}_{y}$ (listen), which carries the information on the chirality, is amplified and recorded. The chiral signal lives until the molecular ensemble rethermalises via collisions with helium atoms in the buffer gas, typically after approximately 5 milliseconds. At this point one cycle is complete and the next one may begin once the field $\mathrm{E}_{x}$ comes back on. As in typical cavity-FTMW spectroscopy, molecules radiate constructively into the cavity mode that is used to polarise the sample. This is an intrinsic feature due to the orthogonal polarisation scheme employed for the experiment. In Fig. 3 (b) we show the averaged free-induction decay (FID) signals for the S- and R-enantiomers of 1,2-propanediol and for a racemic mixture. The S- and R-enantiomers show a clear phase shift of $\pi$ radians, which provides an unambiguous marker for each enantiomer. For the racemic mixture (black trace), the two signals destructively interfere, resulting in zero signal, which is cleaner and more pronounced in the follow-up experiments displayed in Fig. 3 (d) due to advanced instrumentation and a resonant M3WM excitation scheme, which is described in the following.

2.1.2. Fully resonant scheme. The double resonance approach differs from the DC assisted scheme by two key aspects: 1) microwave horn antennas are used to excite and detect, instead of the previously used microwave cavity, which limits the frequencies involved in the M3WM process; 2) the non-resonant (dc) electric field is replaced for RF electrodes, providing a means to resonantly excite the sample at lower frequencies. The double-resonance scheme introduces some noticeable advantages. First, both the drive and the twist transition are now excited in a resonant manner, so that the obtained chiral signal is on the same order of magnitude with respect to the intensity of a "normal" rotational transition. The higher signal-to-noise is easily visible from a comparison of the two FIDs displayed in Figs. 3(b) and 3(d), respectively. Secondly, the detected chiral signal (listen) is at a frequency with no background. Thirdly, RF electrodes are easier to handle and faster to switch on and off than the cavity mirrors used as electrodes in the previous setup given in Fig. 3(a). Finally, since the RF excitation is generated by the same source as the drive frequency, phase stability is locked for the two resonant pulses. A scheme of the experimental setup is shown in Fig. 3(c). Here, two orthogonally-polarized resonant electric fields are applied to generate a third, mutually orthogonal field at their sum frequency. The phase of this induced field changes with enantiomer, and its amplitude gives a direct measure of the enantiomeric excess. The drive field $\mathrm{E}_{c}$ is broadcast via a horn antenna $(\mathrm{A})$ while the twist field $\mathrm{E}_{a}$ acts on the molecular ensemble via the electrodes $(\mathrm{F})$. The induced orthogonal field $\mathrm{E}_{b}$ is captured with the second horn antenna (B). The RF field $\mathrm{E}_{a}$ is created at frequency $\nu_{a}$ when voltage is applied between the electrode $\mathrm{F}$ and the spherical mirror (see Fig. 3 (c)). This double-resonance scheme was employed to measure samples of 1,3-butanediol. The 
(a)
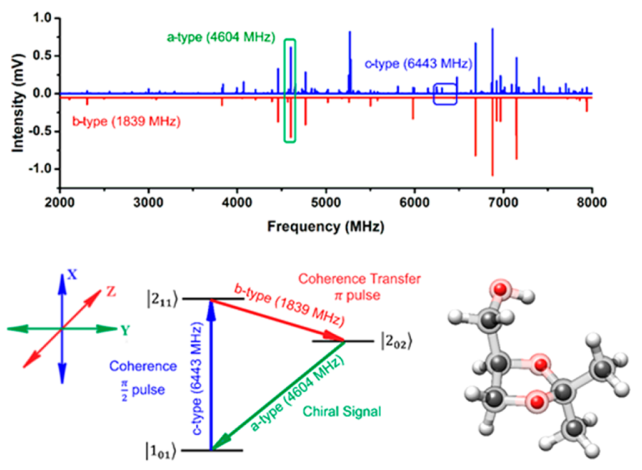

(b)
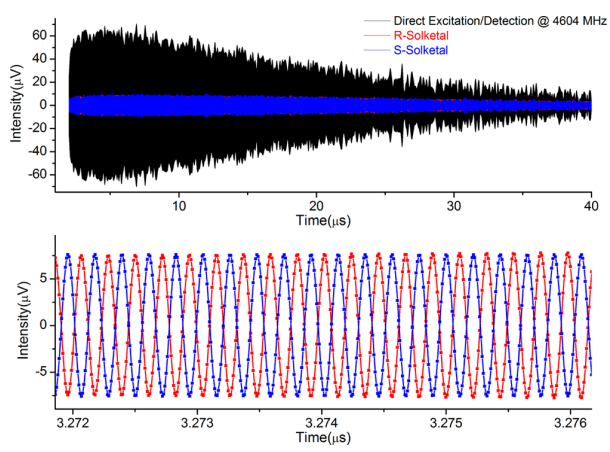

Figure 4

(a) The experimental CP-FTMW spectrum of solketal in the $2-8 \mathrm{GHz}$ frequency region (blue trace) compared to a simulation based on fitted rotational parameters at a rotational temperature of $1.5 \mathrm{~K}$ (red trace). The rotational transitions employed in the three-wave mixing cycle are highlighted in the boxes. The bottom part of this panel schematizes the involved rotational levels and the corresponding polarizations for excitation and detection. A comparison of experimental atom positions with the theoretical structure obtained from calculations is also shown. (b) The enantiomer-dependent FID measured at the listen frequency (4604 MHz). On the top, the directly excited/detected signal (black trace) is compared to the molecular responses for R- (red trace) and S-solketal (blue trace) respectively, to illustrate the differences in amplitudes. The bottom panel shows a zoom-in of the total FID that highlights the $\pi$ radians difference between enantiomers in the time domain. Adapted with permission from Lobsiger et al. (13). Copyright 2015 of the American Chemical Society.

free-induction decay encoding the enantiomer-dependent phase shift is shown in Fig. 3 (d). A net signal of zero arises from a racemic mixture because of destructive interference, which is advantageous, for example for determination of enantiomeric excess.

\subsection{Supersonic expansions}

Microwave three-wave mixing spread further when Shubert et al. applied in 2014 the fully resonant approach to a spectrometer with a supersonic expansion, which is widespread in high-resolution molecular spectroscopy (44). It was thus paramount to transfer the method to a conventional CP-FTMW spectrometer. Shubert et al. modified the broadband spectrometer COMPACT in Hamburg (36) to perform M3WM and measure the absolute configuration and enantiomeric excess of carvone. (10) Only minor modifications are necessary. This study shows that two conformers of the same molecule can be independently and simultaneously investigated for their handedness using M3WM. The high resolution of rotational spectroscopy allows each molecular species to be resonantly probed due to their discrete frequency-resolved rotational states. The excitation pulses can either be applied consecutively or in a co-added manner. Together with the broadband detection capabilities of the spectrometer, analysis of a sample constituted of multiple chiral species is possible. This aspect will be further exploited and discussed in Section 3. Here, and following the scheme given in Fig. 2, a microwave horn antenna broadcasts the drive resonant pulse, while RF electrodes excite the twist resonance. Radiation along the mutually orthogonal orientation is detected via a second horn antenna. The makeup of an ideal pulse sequence relies on 
the following aspects: 1) the transition with the largest population difference (with highest frequency) is chosen for the drive resonance. This is because the listen signal depends on this initial population difference; 2) choosing the transition with the largest dipole moment projection for the listen resonance to maximise the signal-to-noise level for actual detection.

Soon after, in 2015, Brooks Pate and coworkers implemented a second design comprising four horn antennas in prejudice of the RF electrodes. Here, Lobsiger et al.(13) reported an extension of a CP-FTMW spectrometer with the capability of performing M3WM experiments using high-frequency microwave pulses. The four horn scheme allows for a larger frequency range to be explored, in particular for the twist transition, which is limited to a narrow range of radio frequencies in the previous design. A scheme of the experimental setup can be found elsewhere (13). The broadband MW spectrum of solketal is shown in Fig. 4(a). Selected rotational transitions are used to construct the employed energy level scheme using the pertinent polarisations. Fig. 4(b) shows the $\pi$-shifted FIDs for the two enantiomers detected at the listen frequency. In this study, the pulse sequence and timings are explored such as to reach optimal chiral signal. For that, drive, twist, and listen signals are monitored using incremental pulse durations. It is found that maximum coherent molecular listen signal is obtained for Rabi flip angles of $\pi / 2$ and $\pi$, for the drive and twist pulses, respectively, as also outlined in Section 1.1.2.

The fact that M3WM is now implemented in different microwave spectroscopy labs around the world demonstrates the potential of M3WM to differentiate the enantiomers and determine the ee of chiral mixtures prepared in supersonic expansions.

\section{DISENTANGLING CHIRAL MIXTURES}

In the following, we will describe how the combination of broadband rotational spectroscopy in the gas phase and M3WM can be used to disentangle chiral molecules. The broadband capabilities of modern CP-FTMW spectroscopy coupled with M3WM make them a very promising tool for the analysis of complex, chiral mixtures such as essential oils of natural origin, for example. In this section, we will first describe the applications of the combination of CP-FTMW spectroscopy and M3WM to a diastereomeric mixture of menthone, which contains two stereogenic centers, followed by a summary on the study of essential peppermint oil.

\subsection{Menthone}

Many molecules of biological interest contain several stereogenic centers, such as the steroids cholesterol ( 8 stereogenic centers) and estradiol (5 stereogenic centers) or menthone and menthol, with two and three stereogenic centers, respectively. Furthermore, they can come as several conformers. For chiral molecules with more than one stereogenic center, an increasing number of stereoisomers, namely diastereomers and enantiomers, have to be considered. The number of stereoisomers scales with the number of stereogenic centers, $n$, according to $2^{n}$. This can complicate the spectra because the increasing number of stereoisomers potentially leads to dense and widespread spectra with low intensities. Diastereomers differ in their physical and chemical properties. They can be easily distinguished by, for example, their melting and boiling points and their rotational constants. For enantiomer differentiation, however, M3WM or other chirality-sensitive approaches need to be applied. The working principle of M3WM, relying on the opposite signs of the spatial product

Domingos, Pérez and Schnell 
(a)

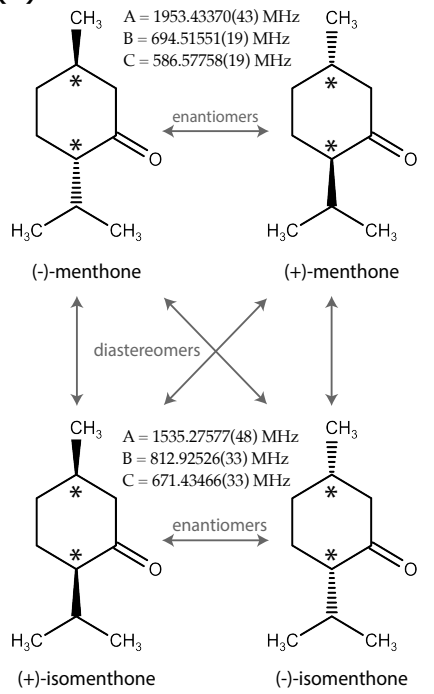

(b)

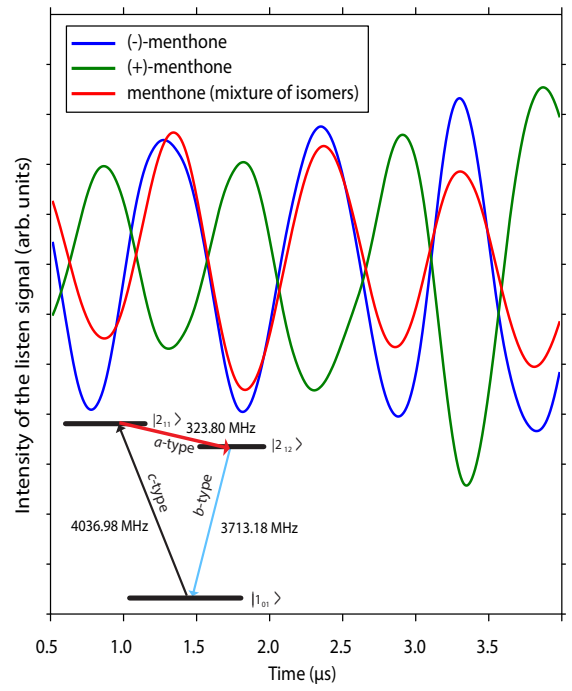

Figure 5

Chiral analysis of menthone. (a) Schematic structures of the four stereoisomers of menthone, which come as two diastereomeric and two enantiomeric pairs. Experimental rotational constants (45) are given for the lowest-energy conformers of menthone (menthone A according to the nomenclature used in Ref. (11)) and isomenthone to illustrate their clear differences. (b) Chiral FID signals obtained for enantio-enriched (-)- and (+)-menthone as well as an uncharacterized mixture of menthone isomers together with the M3WM energy level scheme. Adapted with permission from Shubert et al. (11). Copyright 2014 of Elsevier.

$\vec{\mu}_{a} \cdot\left(\vec{\mu}_{b} \times \vec{\mu}_{c}\right)$ for the two enantiomers, also holds for molecules with multiple stereogenic centers.

Menthone contains two stereogenic centers and thus consists of four stereoisomers, (-)and (+)-menthone as well as (-)- and (+)- isomenthone, where the respective menthone and isomenthone structures are diastereomers with respect to each other and thus can be directly differentiated using their rotational spectra. This is displayed in Fig. 5 using schematics of the molecular structures and the experimentally determined rotational constants for the two diastereomers menthone and isomenthone. (45) The rotational constants differ significantly by up to $20 \%$, resulting in quantitatively and qualitatively different spectra. For menthone, the methyl and the isopropyl groups are in trans arrangement with respect to each other, while for isomenthone they exhibit a cis arrangement. In nature, (-)-menthone is most abundant.

In the following, we focus on the chiral analysis of a commercially available mixture of menthone and isomenthone. By comparison of the M3WM phases obtained for enantiomerenriched samples of menthone with known handedness with the M3WM phase obtained for menthone in the commercial mixture of unknown content, we can identify its handedness. Due to the resonant character, these measurements are not affected by other chiral molecules present in the mixture, including other menthone conformers, for example.

A first series of M3WM experiments was thus performed on enantioenriched samples (with a specified ee of more than 90\%) of (+)- and (-)-menthone. The M3WM cycle 
applied is included in Fig. 5, together with the resulting M3WM signal in form of the FID obtained for the listen transition $J_{\mathrm{K}_{\mathrm{a}} \mathrm{K}_{\mathrm{c}}}: 1_{01} \leftarrow 2_{12}$ at $3713.18 \mathrm{MHz}$ for the enantioenriched samples of (-)-menthone (blue line) and (+)-menthone (green line), as reproduced from Reference (11). Note that the signal is mixed down to $1 \mathrm{MHz}$ for better visibility, which also affects the absolute intensities of the FIDs. As expected, the phases of the M3WM signals differ by $\pi$ radians for $(+)$ - and (-)-menthone resulting from the change in sign of their dipole-moment component products and allows for their differentiation.

The obtained M3WM phases can now be used to obtain information about the uncharacterized menthone mixture of isomers. We applied the same M3WM cycle for menthone to the mixture sample. The resulting FID for the listen transition at $3713.18 \mathrm{MHz}$ is included in Fig. 5 (red line). It agrees well with the FID obtained for $(-)$-menthone under comparable conditions, which clearly indicates that this mixture of isomers is not racemic and $(-)$-menthone is in excess. Note that these measurements can be performed on different samples and days, requiring the vacuum to be broken and the pulsed valve to be removed for changing the sample. This demonstrates the phase stability of the setup as long as no microwave electronic devices are exchanged, which could result in phase changes. By comparison with the chiral signal for the enantioenriched samples, we could thus determine the enantiomer in excess of the menthone mixture of isomers.

\subsection{Peppermint oil}

In this section, we apply the high-resolving power of CP-FTMW spectroscopy and M3WM to the chemical, chiral, and structural analysis of commercially available peppermint oil. Both techniques do not require any prior chemical or chiral separation, which can often be challenging. Peppermint oil is widespread in medicine and aroma therapy. It was investigated in several studies, for example employing gas chromatography coupled with mass spectrometry (GC-MS) aiming at analysing its composition. It was found that the composition of these oils is largely dependent on their origin, chemical treatment and extraction of the oil. The main components of the essential oil from peppermint (Mentha $\mathrm{x}$ piperita L.) are menthol $(40.7 \%)$ and menthone $(23.4 \%)$, based on the age of the plants, and GCMS analysis. Further components are menthyl acetate, 1,8 -cineole, limonene, $\beta$-pinene and $\beta$-caryophyllene. (46)

Fig. 6 shows three sections of the broadband rotational spectrum of peppermint oil obtained with the COMPACT spectrometer. The fingerprint character of rotational spectroscopy allows us to directly and definitely identify nine different terpene molecules as well as four more conformers based on their rotational constants. These are also included in Fig. 6. Clear intensity differences for the rotational transitions of the various components are observed. Eucalyptol is found to be the most intense species, followed by menthol, menthone, and isomenthone. For $\beta$-pinene, limonene, pulegone, and menthyl acetate the rotational transitions are less intense by approximately one order of magnitude. The observed transition strengths for such a measurement of a multi-component sample like peppermint oil depend on a number of factors. In the weak-pulse limit of CP-FTMW spectroscopy, the transition intensities depend on the strength of the excitation field, the square of the transition dipole moments of the individual molecules as well as the number of molecules interacting with the microwave pulse. Before supersonically expanding the molecular sample into the microwave interaction zone, we heat the samples to increase the vapour pressures of the constituents. This results in molecule- and mixture-dependent vapour pressures and 

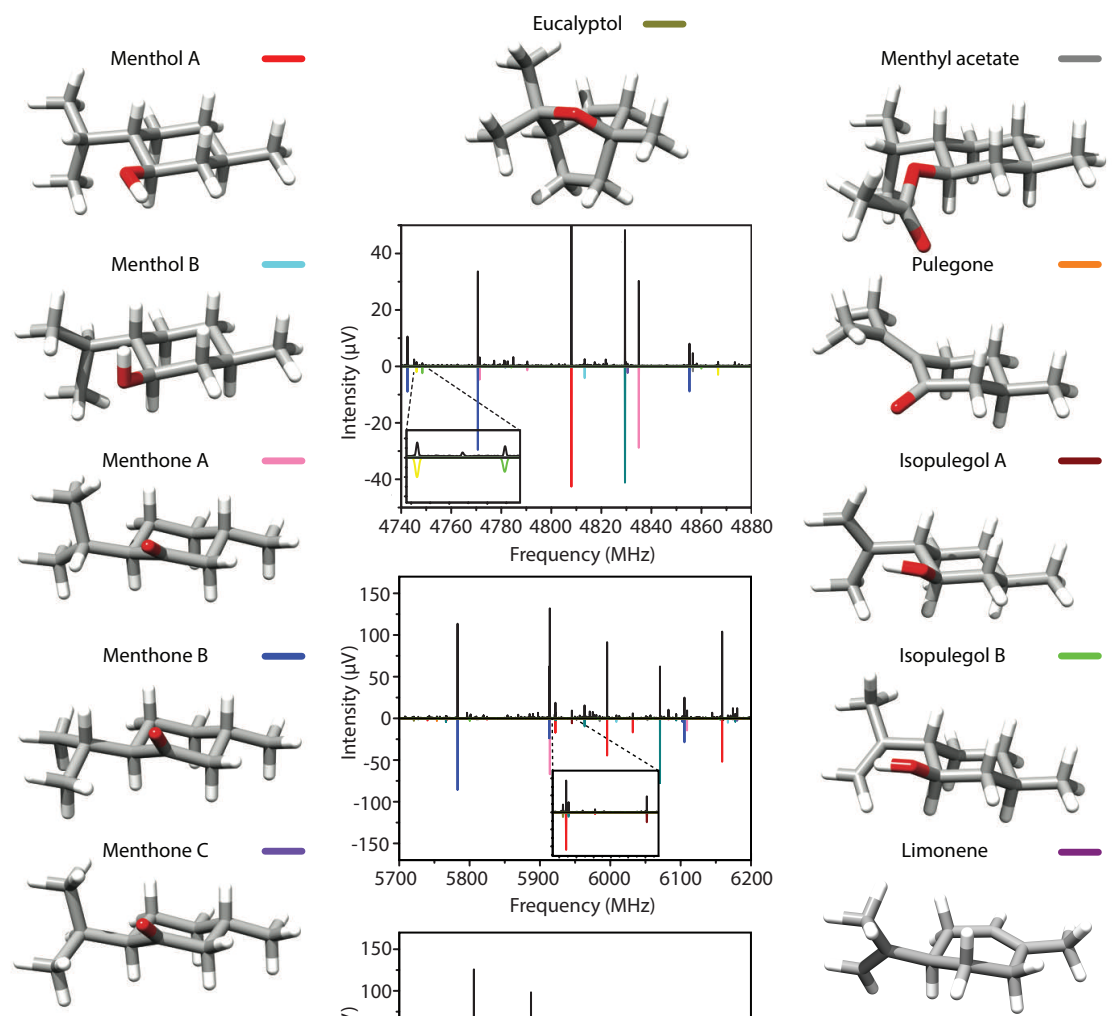

Isopulegol B
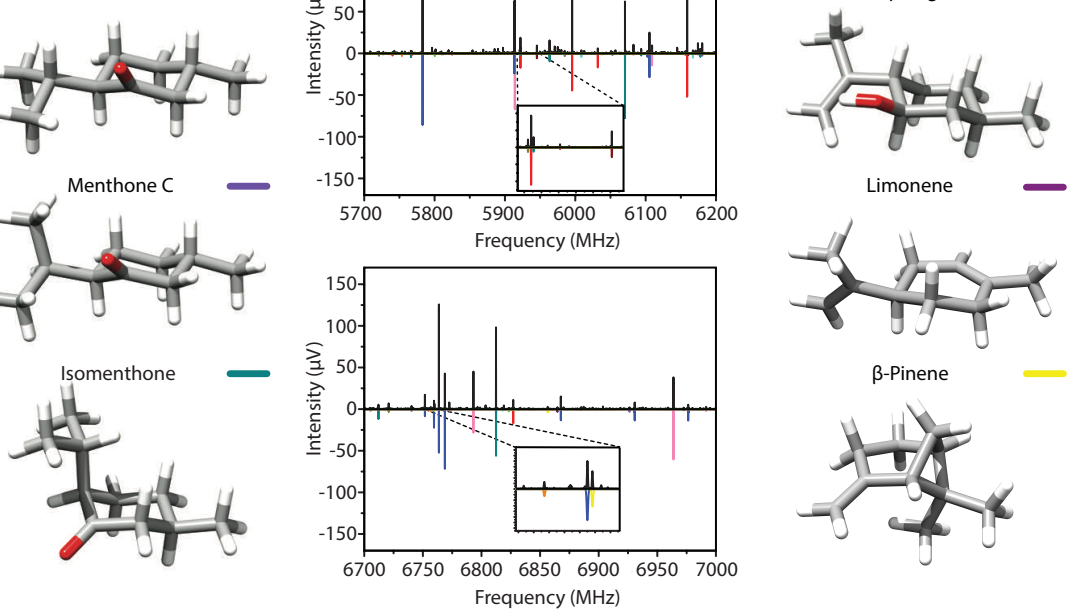

Figure 6

Three sections of the broadband rotational spectrum of commercially available peppermint oil. The upper traces (black) show the experimental results, while the lower traces show simulations of rotational spectra of several terpene molecules based on their experimental rotational constants. The different colours indicate the various molecules that are structurally related. Insets: zooms of the broadband spectrum revealing less abundant components.

thus number densities for the different constituents. Some species will be vaporised more efficiently than others, potentially resulting in stronger signals. Furthermore, there is an instrument effect on the transition intensities. For example, the output power of the TWT amplifier is frequency dependent over the range of the $2-8 \mathrm{GHz}$ chirp, providing around $300 \mathrm{~W}$ at the edges and up to $600 \mathrm{~W}$ output power at the centre of the frequency band, according to specifications. In principle, such instrument effects can be corrected for with an instrument function. Here, however, we focus on a more qualitative analysis of the oil components and their chirality. 
Transfer pulse: microwave pulse resonant with the listen transition.

Population enrichment: the term refers to the increase in population of a selected rotational state of a specific enantiomer. Which enantiomer is promoted to a higher rotational state is defined by the phase of the transfer pulse.
Most of the more prominent components of peppermint oil are chiral and can be expected to occur enantioenriched because of the natural origin of the essential oil. From an analytical point of view, the analysis of such a sample can be extremely challenging. However, as discussed above, M3WM relies on the high-resolution fingerprint character of rotational spectroscopy. It is a resonant, non-linear, and coherent technique, and as such, a signal can be recorded for specific chiral species simultaneously without being perturbed by other (chiral) species in the sample, as we have for example shown for two conformers of carvone.(10)

As a proof-of-principle experiment, we performed M3WM for menthone and its diastereomer isomenthone in the oil. For menthone, we use the M3WM cycle displayed in Fig. 5, and identify (-)-menthone as the excess enantiomer in peppermint oil by comparison of the respective M3WM phases obtained for samples with known excess enantiomer, similar to the experiments shown in Fig. 6 for the uncharacterised menthone mixture. Isomenthone is not available for purchase in an enantioenriched form. The chiral signature in M3WM depends on the sign of the product of the transition dipole moment components $\mu_{a}, \mu_{b}$, and $\mu_{c}$. Using the phases obtained for (-)-menthone as a standard, we can extract the absolute configuration of isomenthone via comparison of the product of the transition dipole-moment components and the observed phases of the listen transitions. The results indicate that we have (+)-isomenthone in the sample, which is in agreement with the outcome of GC-MS measurements reported before. (47) These results nicely demonstrate the applicability of M3WM to complex chiral samples like commercially available essential oils.

\section{SHUFFLING ENANTIOMERS WITH TAILORED MICROWAVE FIELDS}

A challenging and longstanding goal of chiral experiments is to implement fast and reliable methods for purifying chiral samples solely through optical means. The natural next step in the development of M3WM experiments is thus to demonstrate its ability to enrich or even purify a chiral sample. Schemes to achieve enantio-enrichment via cyclic population processes have been proposed as a way to isolate a particular enantiomer in a quantum state, however in the optical regime. $(48,49)$ Based on the M3WM approach, enantiomer-selective population enrichment has been recently demonstrated in both a buffer-gas cell (50) and, soon after, a supersonic expansion (51) using tailored microwave fields.

As it has been previously stated, M3WM experiments comprise of two microwave pulses that coherently generate the chiral signature using a closed cycle of dipole-allowed rotational transitions (Fig. 2). In order to achieve enantiomer-selective population enrichment, an additional microwave pulse, at the same frequency as the chiral signal, the listen transition, is applied. This is illustrated in Fig. 7 where the implementation of the population transfer scheme using the example of carvone is shown. The experiment involves simultaneous excitation of three rotational transitions.

While the transitions $\left|3_{12}\right\rangle \rightarrow\left|2_{02}\right\rangle(5557.91 \mathrm{MHz})$ and $\left|3_{12}\right\rangle \rightarrow\left|3_{13}\right\rangle(459.75 \mathrm{MHz})$ correspond to the drive and twist transitions, respectively, the cycle is closed by applying a pulse at the frequency of $\left|3_{13}\right\rangle \rightarrow\left|2_{02}\right\rangle(5098.12 \mathrm{MHz})$, the transfer pulse. (Note that in the pure M3WM scheme, this corresponds to the listen transition. The difference between "normal" M3WM for enantiomer differentiation and chiral analysis and the enantiomerselective population transfer as described in the present section is that the listen transition is actively excited in a phase-dependent manner.)

To generate population transfer, two factors need to be considered. First, the transfer 
(a)
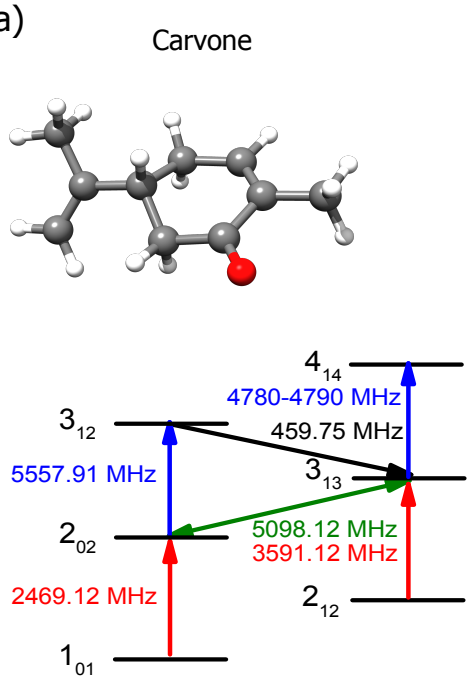

(b)
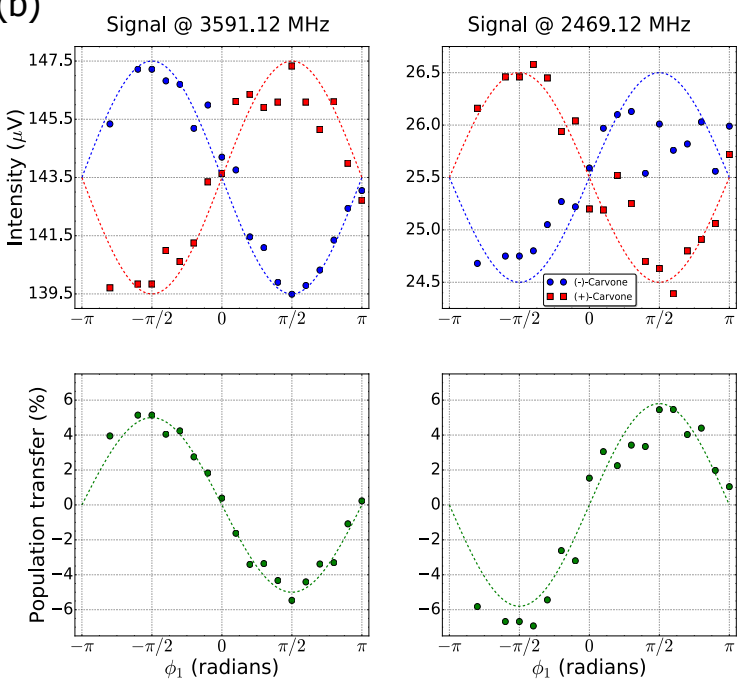

Figure 7

(a) Energy level schematic of carvone exploited to achieve enantiomer-selective population enrichment. The $\left|3_{12}\right\rangle \rightarrow\left|2_{02}\right\rangle(5557.91 \mathrm{MHz})$ and $\left|3_{12}\right\rangle \rightarrow\left|3_{13}\right\rangle(459.75 \mathrm{MHz})$ rotational transitions correspond to the drive and twist transitions, respectively, while the pulse at $\left|3_{13}\right\rangle \rightarrow\left|2_{02}\right\rangle(5098.12 \mathrm{MHz})$ is the transfer pulse. Careful scan of the phase of this transfer pulse leads to the $\left|2_{02}\right\rangle$ and $\left|3_{13}\right\rangle$ rotational states being populated or depopulated with the desired enantiomer. The effect is monitored on the intensity of two probe transitions shown in red. (b) Experimental intensity of the two probe transitions at $3591.12 \mathrm{MHz}\left(\left|3_{13}\right\rangle \rightarrow\left|2_{12}\right\rangle\right)$ and 2469.12 $\mathrm{MHz}\left(\left|2_{02}\right\rangle \rightarrow\left|1_{01}\right\rangle\right)$. The blue trace is the observed signal for $(-)$-carvone while the red trace shows the $(+)$-carvone response. As shown, they exhibit opposite behaviour. The bottom panels show the normalized enantiomer-selective population enrichment in $\left|3_{13}\right\rangle$ and $\left|2_{02}\right\rangle$ with respect to the direct excitation of the same transition when the loop of transitions is turned off. Figures adapted with permission from Pérez et al. (51). Copyright 2017 of Wiley-VCH.

pulse needs to be applied in the third mutually orthogonal direction with respect to the drive and twist pulses. Second, the relative phase of all three pulses is critical to achieve the desired enrichment. In the example in Fig. 7, the phase of the transfer pulse was systematically varied from $-\pi$ to $+\pi$ while keeping the other two pulses at the same relative phase. Under these conditions and when the optimal relative phase is selected, one of the enantiomers is selectively transferred to either $\left|3_{13}\right\rangle$ or $\left|2_{02}\right\rangle$ while the other enantiomer exhibits the opposite behaviour.

Of particular importance here is that the achieved population enrichment will be limited by the residual thermal population in a particular rotational level, and the transferred population may be small compared to it. This may cause the effect to be small, especially for closely spaced rotational transitions. In order to improve this, a fast chirp fulfilling the Rapid Adiabatic Passage (52) (RAP) regime can be applied to selectively transfer the undesired thermal population to higher, more unoccupied rotational levels. This was done in the carvone study, by applying a RAP pulse around the $\left|4_{14}\right\rangle \rightarrow\left|3_{13}\right\rangle$ rotational transition with a bandwidth of $10 \mathrm{MHz}$. As mentioned, this three-pulse scheme renders a population enrichment or depopulation in the rotational levels of choice of one of the enantiomers. 
Since the intensity of a rotational transition depends on the population difference between rotational levels (amongst other factors), this induced population can be then probed using a fourth rotational transition as a readout with the only requirement that it is connected to either $\left|3_{13}\right\rangle$ or $\left|2_{02}\right\rangle$. The polarisation direction of this probe transition is not relevant, and thus it can be performed in any of the three polarizations.

Panel (b) in Fig. 7 shows the phase-dependent behaviour of two rotational transitions connected to $\left|3_{13}\right\rangle$ and $\left|2_{02}\right\rangle$, respectively. The magnitude of the observed signal varies sinusoidally with the phase of the transfer pulse. In both cases, corresponding to the two enantiomers, the transition used as a probe has the upper level involved in the closed population transfer loop. This leads to an opposite response for the same enantiomer when the intensity is monitored, which clearly shows that the enantiomers are being selectively transferred. The achieved population enrichment can be then quantified by normalising this phase-dependent signal with respect to the observed intensity of the probe transition when the whole drive-twist-transfer loop is turned off. These results are shown on the bottom of Fig. 7(b), revealing that a $6 \%$ enrichment was achieved. This enantiomeric enrichment is one order of magnitude larger than that reported in a buffer-gas cell experiment. (50) As stated above, the observed contrast is largely dependent on the thermal distribution in the rotational level manifold. While in a buffer-gas cell it is assumed that the rotational temperature is on the order of 7-10 K, the lower rotational temperature of a molecular beam, 1-2 K, enhances the observed contrast. Moreover, the use of RAP pulses also favours larger enrichments as the residual population in the target rotational level is to a great extent reduced. Combined with additional electrostatic deflectors $(53,54)$, laser methods, or even additional microwave pulse schemes, the possibility for more sophisticated approaches to physically separate or selectively deplete one of the enantiomers comes into play. As long as further manipulation occurs within the appropriate timescales, i.e., before thermal redistribution takes place, the results of this population enrichment method pave the way towards developing more sophisticated tools for chiral analysis and have the potential to usher into a new stage of control and manipulation of chiral samples.

\section{OUTLOOK}

It is less than a hand-full of years after the first experimental demonstration of enantiomerspecific detection of chiral molecules using microwave spectroscopy, and the new concept is still gaining momentum. The significance of a new, conceptually distinct approach to measuring chirality of molecules opens up a plethora of opportunities for a broad scope of applications, both of fundamental and of applied nature. Samples of quantum-stateand enantiomer-selected chiral molecules can be prepared for fundamental physics experiments, and for a wide range of potential analytical applications such as spatial separation of enantiomers and chiral purification. With the implementation of broadband excitation schemes, microwave spectroscopy has become a fast, versatile technique with the potential to compete on equal footing with other more established structure determination tools such as NMR spectroscopy.

The resonant nature of the technique establishes a robust means to detect individual chiral components within samples containing a large number of species, as shown in Section 3, inspiring the sought of further applications for the analytical sciences. For example, trace analysis of small amounts of chemical components in complex mixtures are highly desirable in the pharmaceutical and forensic sciences. Intrinsic to the high resolution achieved 
in broadband rotational spectroscopy, complex mixtures of naturally occurring oils can be disentangled and its constituents identified, not only for their conformation, but their handedness as well. As we have discussed here in the context of peppermint oil, the fingerprint character of rotational spectroscopy allows for the definite assignment of structurally very similar molecules. Despite the presence of several chiral species simultaneously, the handedness of the individual chiral components can be determined with M3WM because it is highly molecule-specific. The chiral signals do not interfere with each other because they are excited and probed using different rotational frequencies. However, determination of the absolute configuration using M3WM without any prior knowledge is not satisfactorily solved to date, because it requires the exact determination of the absolute phase of the chiral FID. Due to the high frequencies at play here, an intelligent solution for this obstacle is still in the making. So far, at the current stage, the absolute configuration can be determined via comparison with samples of known handedness.

While the "absolute phase" problem is not solved, an elegant alternative comes into play: the chiral tagging method. This method makes use of the fact that molecular complexes comprising chiral molecules form pairs of diastereomers, which can be easily differentiated based on their rotational spectra, similar to the diastereomers of menthone displayed in Fig. 5. For chiral tagging, the principle is the same. A chiral sample containing enantiomers of a certain handedness and with a potentially unknown ee will be complexed with another chiral molecule, the chiral tag, which is added with known ee. The chiral tag will now bind to the enantiomers of the molecules of interest via intermolecular interactions undergoing the formation of diastereomers: An R-enantiomer chiral tag will form an RRdiastereomer with the R-enantiomer of the sample of interest and an RS-diastereomer with the corresponding S-enantiomer. These two complexes have different rotational constants and can thus be differentiated in a straightforward manner. By comparison with quantumchemical calculations, for example, it provides direct access to the handedness of the chiral molecule of interest. Moreover, the transition intensities can provide quantitative information on the ee. This procedure is a well-known chemical principle for differentiating and separating enantiomers, also exploited in organic synthesis, for example. Using this trick, the challenge of differentiating and assigning the enantiomers of chiral molecules has been transferred to the much easier task of analysing diastereomers.

An emerging trend is set not only to establish M3WM as a fast and robust means to determine the absolute configuration and enantiomeric excess of chiral molecules, but to also exploit the possibilities with regards to advanced experiments with chiral molecules beyond chiral analysis. A future goal for the research on chiral molecules with tailored microwave fields is to provide a microscopic understanding of chirality, and to study, control, and manipulate chirality at the molecular level in the gas phase.

One of the grand challenges of chiral molecule research is to achieve enantiomer separation and chiral purification, which is an important goal in its own right, but also the basis for other advanced experiments requiring molecular control. A first step in that direction is discussed in Section 4 where experiments were discussed that show how tailored microwave pulses can be used to not only read out the chirality of a molecule but also to promote separation.(50, 51) With the concept of coherent state-specific enrichment now being experimentally observed, many applications of such coherent control come to mind. It can be envisioned that, using state-selective laser excitation or exploiting the state selectivity of the Stark effect using inhomogeneous electric fields, for example, this population difference for the enantiomers will be transformed into spatial separation. It will thus become possible 
to generate packets of selected enantiomers in a single quantum state, which are unique and outstanding starting points for new experiments, such as chirality-dependent collisions and precision measurements aiming at determining the frequency difference between the enantiomers. The preparation of chiral molecules in rotational states of choice introduces the required control to exploit for example measurements of parity violation in chiral molecules.(55-58)

"The unexpected demonstration of a conceptually new form of chiroptical spectroscopy makes this work a landmark in the 200-year-old history of optical activity in chemistry." L. A. Nafie (59)

\section{DISCLOSURE STATEMENT}

The authors are not aware of any affiliations, memberships, funding, or financial holdings that might be perceived as affecting the objectivity of this review.

\section{ACKNOWLEDGMENTS}

We acknowledge Alvin Shubert, David Schmitz, Dave Patterson, John Doyle, Anna Krin and Amanda Steber for their contributions to the work presented in this review and for insightful discussions. This work has been supported by the excellence cluster "The Hamburg Centre for Ultrafast Imaging - Structure, Dynamics and Control of Matter at the Atomic Scale" of the Deutsche Forschungsgemeinschaft (DFG) and via DFG grant number SCHN1280/4-1. S.R.D. and C. P. acknowledge a Postdoctoral Research Fellowship from the Alexander von Humboldt Foundation.

\section{LITERATURE CITED}

7. Proof-of-concept experiment of chiral-sensitive microwave spectroscopy in a cooled buffer gas: single resonance scheme.

8. The first experimental demonstration of M3WM in a cooled buffer gas: double resonance scheme.
1. Pasteur L. 1948. Sur les relations qui peuvant exister entre la forme crystalline, la composition chimique et le sens de la polarization rotatoire. Ann. Chim. Phys. 24:442-459

2. Sanganyado E, Lu Z, Fu Q, Schlenk D, Gan J. 2017 Chiral pharmaceuticals: A review on their environmental occurrence and fate processes. Water Research 124:527:542

3. Berova N, Nakanishi K. 2000. Circular dichroism: Principles and applications. John Wiley \& Sons

4. Nafie LA. 2011. Vibrational optical activity: Principles and applications. John Wiley \& Sons, Ltd, Chichester, UK

5. Nafie LA, Keiderling TA, Stephens PJ. 1976. Vibrational circular dichroism. J. Am. Chem. Soc. 98:2715-2723

6. Barron LD. 2004. Molecular light scattering and optical activity. Cambridge Univ. Press: Cambridge, UK

7. Patterson D, Schnell M, Doyle JM. 2013. Enantiomer-specific detection of chiral molecules via microwave spectroscopy. Nature 497:475

8. Patterson D, Doyle JM. 2013. Sensitive chiral analysis via microwave three-wave mixing. Phys. Rev. Lett. 111:023008

9. Grabow JU. 2013. Fourier transform microwave spectroscopy: Handedness caught by rotational coherence. Angew. Chem. Int. Ed. 52:11698-11700

10. Shubert VA, Schmitz D, Patterson D, Doyle JM, Schnell M. 2014. Identifying enan- 
tiomers in mixtures of chiral molecules with broadband microwave spectroscopy. Angew. Chem. Int. Ed. 4:1152-1155

11. Alvin Shubert V, Schmitz D, Schnell M. 2014. Enantiomer-sensitive spectroscopy and mixture analysis of chiral molecules containing two stereogenic centers - microwave three-wave mixing of menthone. J. Mol. Spec. 300:31-36

12. Patterson D, Schnell M. 2014. New studies on molecular chirality in the gas phase: enantiomer differentiation and determination of enantiomeric excess. Phys. Chem. Chem. Phys. 16:1111411123

13. Lobsiger S, Pérez C, Evangelisti L, Lehman KK, Pate BH. 2015. Molecular structure and chirality detection by Fourier transform microwave spectroscopy. J. Phys. Chem. Lett. 6:196?200

14. Shubert VA, Schmitz D, Medcraft C, Krin A, Patterson D, et al. 2015. Rotational spectroscopy and three-wave mixing of 4-carvomenthenol: A technical guide to measuring chirality in the microwave regime. J. Chem. Phys. 142:214201

15. Shubert VA, Schmitz D, Pérez C, Medcraft C, Krin A, et al. 2016. Chiral analysis using broadband rotational spectroscopy. J. Phys. Chem. Lett. 7:341-350

16. Horsch P, Urbasch G, Weitzel KM. 2012. Analysis of chirality by femtosecond laser ionization mass spectrometry. Chirality 24:684-690

17. Lux C, Wollenhaupt M, Bolze T, Liang Q, Kohler J, et al. 2012. Circular dichroism in the photoelectron angular distributions of camphor and fenchone from multiphoton ionization with femtosecond laser pulses. Angew. Chem. Int. Ed. 20:5001-5005

18. Garcia GA, Nahon L, Daly S, Powis I. 2013. Vibrationally induced inversion of photoelectron forward-backward asymmetry in chiral molecule photoionization by circularly polarized light. Nature Communications 4:2132

19. Janssen MHM, Powis I. 2014. Detecting chirality in molecules by imaging photoelectron circular dichroism. Phys. Chem. Chem. Phys. 16:856-871

20. Pitzer M, Kunitski M, Johnson AS, Jahnke T, Sann H, et al. 2013. Direct determination of absolute molecular stereochemistry in gas phase by coulomb explosion imaging. Science 341:10961100

21. Herwig P, Zawatzky K, Grieser M, Heber O, Jordon-Thaden B, et al. 2013. Imaging the absolute configuration of a chiral epoxide in the gas phase. Science 342:1084-1086

22. Hansen N, Wullenkord J, Obenchain DA, Graf, Kohse-Höinghaus K, Grabow J.-U. 2016. Microwave spectroscopy detection of flame-sampled combustion intermediates. RSC Adv. 7:37867

23. Park BG, Field R. 2016. Perspective: The first ten years of broadband chirped pulse Fourier transform microwave spectroscopy. J. Chem. Phys. 144:200901

24. Brown GG, Dian BC, Douglass KO, Geyer SM, Shipman ST, Pate BH. 2008. A broadband fourier transform microwave spectrometer based on chirped pulse excitation. Rev. Sci. Instrum. 79:053103

25. Domingos SR, Pérez C, Medcraft C, Pinacho P, Schnell M. 2016. Flexibility unleashed in acyclic monoterpenes: conformational space of citronellal revealed by broadband rotational spectroscopy. Phys. Chem. Chem. Phys. 18:16682-16689

26. Seifert NA, Finneran IA, Pérez C, Zaleski DP, Neill JL, Steber AL, Suenram RD, Lesarri A, Shipman ST, Pate BH. 2015. AUTOFIT, an automated fitting tool for broadband rotational spectra, and applications to 1-hexanal. J. Mol. Spectrosc. 312:13-21

27. Pérez C, Muckle MT, Zaleski DP, Seifert NA, Temelso B, et al. 2012. Structures of cage, prism, and book isomers of water hexamer from broadband rotational spectroscopy. Science 336:897901

28. Pérez C, Zaleski DP, Seifert NA, Temelso B, Shields GC, et al. 2014. Hydrogen bond cooperativity and the three-dimensional structures of water nonamers and decamers. Angew. Chem. Int. Ed. 53:14368-14372

29. Steber AL, Harris BJ, Neill JL, Pate BH. 2012. An arbitrary waveform generator based chirped
10. The first

measurement of

M3WM in a

supersonic jet

experiment.

14. Practical guide

to measuring

M3WM.
24. The beginning of CP-FTMW spectroscopy. 
36. Technical report of the COMPACT spectrometer.

37. Theoretical formulation of $3 \mathrm{WM}$ in chiral molecules.

50. Proof of concept of state-selective population transfer in chiral molecules. pulse fourier transform spectrometer operating from 260 to 295ghz. J. Mol. Spectrosc. 280:3?-10

30. Zaleski DP, Seifert NA, Steber AL, Muckle MT, Loomis RA, et al. 2013. Detection of ecyanomethanimine toward sagittarius $\mathrm{b} 2(\mathrm{n})$ in the green bank telescope primos survey. ApJL 765:L10

31. Medcraft C, Wolf R, Schnell M. 2014. High-resolution spectroscopy of the chiral metal complex [cpre(ch3)(co)(no)]: A potential candidate for probing parity violation. Angew. Chem. Int. Ed. 53:11656-11659

32. Bittner DM, Zaleski DP, Tew DP, Walker NR, Legon AC. 2016. Highly unsaturated platinum and palladium carbenes ptc3 and pdc3 isolated and characterized in the gas phase. Angew. Chem. Int. Ed. 55:3768-3771

33. Zaleski DP, Stephens SL, Walker NR. 2014. A perspective on chemistry in transient plasma from broadband rotational spectroscopy. Phys. Chem. Chem. Phys. 16:25221-25228

34. Kidwell NM, Vaquero-Vara V, Ormond TK, Buckingham GT, Zhang D, et al. 2014. Chirpedpulse fourier transform microwave spectroscopy coupled with a flash pyrolysis microreactor: Structural determination of the reactive intermediate cyclopentadienone. J. Phys. Chem. Lett. 5:2201-2207

35. Prozument K, Colombo AP, Zhou Y, Park GB, Petrović VS, et al. 2011. Chirped-pulse millimeter-wave spectroscopy of rydberg-rydberg transitions. Phys. Rev. Lett. 107:143001

36. Schmitz D, Alvin Shubert V, Betz T, Schnell M. 2012. Multi-resonance effects within a single chirp in broadband rotational spectroscopy: The rapid adiabatic passage regime for benzonitrile. J. Mol. Spec. 280:77-84

37. Hirota E. 2012. Triple resonance for a three-level system of a chiral molecule. Proc. Jpn Acad. Ser. B Phys. Biol. Sci. 88:120-128

38. Blum K. 1981. Density matrix theory and applications. Plenum Press, New York

39. Crabtree KN, Martin-Drumel MA, Brown GG, Gaster SA, Hall TM, McCarthy MC. 2016. Microwave spectral taxonomy: A semi-automated combination of chirped-pulse and cavity fourier-transform microwave spectroscopy. J. Chem. Phys. 144:124201

40. Martin-Drumel MA, McCarthy MC, Patterson D, McGuire BA, Crabtree KN. 2016. Automated microwave double resonance spectroscopy: A tool to identify and characterize chemical compounds. J. Chem. Phys. 144:124202

41. Hutzler NR, Lu HI, Doyle JM. 2012 The Buffer Gas Beam: An Intense, Cold, and Slow Source for Atoms and Molecules Chem. Rev. 112(9):4803-4827

42. Drayna GK, Hallas C, Wang K, Domingos SR, Eibenberger S, et al. 2016. Direct time-domain observation of conformational relaxation in gas-phase cold collisions. Angew. Chem. Int. Ed. 55:4957-4961

43. Spaun B, Changala PB, Patterson D, Bjork BJ, Heckl OH, et al. 2016. Continuous probing of cold complex molecules with infrared frequency comb spectroscopy. Nature 533:517?-520

44. Quack M, Merkt F, eds. 2011. Handbook of high resolution spectroscopy. Wiley

45. Schmitz D, Shubert VA, Betz T, Schnell M. 2015. Exploring the conformational landscape of menthol, menthone, and isomenthone: a microwave study. Frontiers in Chemistry 3:15

46. Rohloff J. 1999 Monoterpene Composition of Essential Oil from Peppermint (Mentha x piperita L.) with Regard to Leaf Position Using Solid-Phase Microextraction and GAs Chromatography/Mass Spectrometry Analysis. J. Agric. Food Chem. 47:3782:3786

47. Menary RC, Garland SM. 1999 Authenticating Essential Oil Flavours and Fragrances. Rural Industries Research and Development Corporation (RIRDC) 99:125

48. Shapiro M, Frishman E, Brumer P. 2000. Coherently controlled asymmetric synthesis with achiral light. Phys. Rev. Lett. 84:1669-1672

49. Král P, Shapiro M. 2001. Cyclic population transfer in quantum systems with broken symmetry. Phys. Rev. Lett. 87:183002

50. Eibenberger S, Doyle J, Patterson D. 2017. Enantiomer-specific state transfer of chiral molecules. Phys. Rev. Lett. 118:123002 
51. Pérez C, Steber AL, Domingos SR, Krin A, Schmitz D, Schnell M. 2017. Coherent enantiomer-selective population enrichment using tailored microwave fields. Angew. Chem. Int. Ed. 56:12512-12517

52. Malinovsky V, Krause J. 2001. General theory of population transfer by adiabatic rapid passage with intense, chirped laser pulses. Eur. Phys. J. D 14:147-155

53. Filsinger F, Küpper J, Meijer G, Hansen JL, Maurer J, et al. 2009. Pure samples of individual conformers: The separation of stereoisomers of complex molecules using electric fields. Angew. Chem. Int. Ed. 48:6900-6902

54. Chang YP, Horke DA, Trippel S, Küpper J. 2015. Spatially-controlled complex molecules and their applications. Int. Rev. Phys. Chem. 34:557-590

55. Quack M, Stohner J, Willeke M. 2008. High-resolution spectroscopic studies and theory of parity violation in chiral molecules. Annu. Rev. Phys. Chem. 59:741-769

56. Quack M. 2002. How important is parity violation for molecular and biomolecular chirality? Angew. Chem. Int. Ed. 41:4618?-4630

57. Quack M, Stohner J, Willeke M. 2008. High-resolution spectroscopic studies and theory of parity violation in chiral molecules. Ann. Rev. Phys. Chem. 59:741-769

58. Darquié B, Stoeffler C, Shelkovnikov A, Daussy C, Amy-Klein A, et al. 2010. Progress toward the first observation of parity violation in chiral molecules by high-resolution laser spectroscopy. Chirality 22:870-884

59. Nafie LA. 2013. Physical chemistry: Handedness detected by microwaves. Nature 497:446?-448

51. First demonstration of population enrichment in a supersonic jet experiment. 